\title{
Control of Hovering Spacecraft Near Small Bodies: Application to Asteroid 25143 Itokawa
}

\author{
Stephen B. Broschart* and Daniel J. Scheeres ${ }^{\dagger}$ \\ University of Michigan, Ann Arbor, Michigan 48109-2140
}

\begin{abstract}
The small gravitational forces associated with a minor celestial body, such as an asteroid or comet nucleus, allow visiting spacecraft to implement active control strategies to improve maneuverability about the body. One form of active control is hovering, where the nominal accelerations on the spacecraft are canceled by a nearly continuous control thrust. We investigate the stability of realistic hovering control laws in the body-fixed and inertial reference frames. Two implementations of the body-fixed hovering thrust solution are numerically tested and compared with an analytical stability result presented in the previous literature. The perturbation equations for inertial frame hovering are presented and numerically simulated to identify regions of stability. We find that body-fixed hovering can be made stable inside a region roughly approximated by the body's resonance radius and inertial frame hovering is stable in all regions outside the resonance radius. A case study of hovering above Asteroid (25143) Itokawa, target of the Hayabusa mission, is also presented.
\end{abstract}

\section{Introduction}

A T the Japanese, European, and U.S. space agencies, missions currently exist to send spacecraft to explore minor celestial objects such as asteroids, comets, and small planetary satellites. It is suspected that the elemental composition of these objects, relatively uncorrupted by time, may offer scientists insight into the early history of our solar system. Thus, successful missions to such objects are of critical importance to the ongoing goal of understanding the formation and evolution of our solar system.

These objects are generally quite small compared to the planets and moons that have been the targets of most space missions to date. Because of their small size and correspondingly weak gravitational pull, they are also usually quite irregular in shape. These attributes present great challenges to the traditional "orbiter-type" control strategies used to date. Orbital trajectories about small, irregularly shaped bodies are generally quite complex and nonperiodic. ${ }^{1}$ Further, stability for close orbits above many small bodies is guaranteed only for a limited range of latitudes, ${ }^{2}$ possibly preventing study of relevant regions of the body's surface. Also, because the gravitational forces are small, solar radiation pressure becomes an important perturbing force, driving orbits to instability for objects smaller than a particular size. ${ }^{3}$

These problems with orbital control strategies for missions to small bodies necessitate a paradigm shift in the way we control spacecraft. One solution that has been proposed is hovering. ${ }^{4,5}$ This active control strategy uses thrusters in a nearly continuous manner to null the nominal accelerations on the spacecraft, creating an equilibrium point at the desired position. This approach is feasible for operating near a small body because of the relatively weak gravitational forces involved. Unlike orbital control approaches, hovering control can be used to explore any size of body at any latitude, limited only by fuel restrictions. The perturbing forces of solar radiation pressure and solar gravity are also less of a concern in hovering, because these forces can be actively nulled by the hovering controller.

Received 15 July 2003; revision received 7 January 2004; accepted for publication 12 January 2004. Copyright (C) 2004 by Stephen B. Broschart. Published by the American Institute of Aeronautics and Astronautics, Inc., with permission. Copies of this paper may be made for personal or internal use, on condition that the copier pay the $\$ 10.00$ per-copy fee to the Copyright Clearance Center, Inc., 222 Rosewood Drive, Danvers, MA 01923; include the code 0731-5090/05 \$10.00 in correspondence with the CCC.

${ }^{*}$ Graduate Student, 2016 FXB, Department of Aerospace Engineering; sbroscha@umich.edu.

'Associate Professor, 3048 FXB, Department of Aerospace Engineering; scheeres@umich.edu. Associate Fellow AIAA.
In this paper, we investigate two types of hovering: hovering in the small-body-fixed frame and in the inertial frame. Hovering in the body-fixed frame would be ideal for a spacecraft operating very close to the body's surface. Because body-fixed hovering fixes the spacecraft's position relative to the body, this control could be advantageous for obtaining high-resolution measurements of a particular area on the body's surface. Body-fixed hovering also simplifies descent and ascent maneuvers that may be necessary for a sample return mission. A spacecraft visiting a small body may also be interested in hovering in the inertial frame, where the small body rotates beneath the spacecraft. Inertial-frame hovering may be ideal for a spacecraft mapping a body's surface or for use as a holding orbit from which to stage other maneuvers. For hovering to be a viable mission option, it must be shown to be stable and robust over a range of situations and implementable under realistic control constraints. This paper uses analytic and numerical analysis to identify regions of stable operation for a spacecraft subject to hovering control.

We begin by formulating the solutions of the two-body problem corresponding to body-fixed and inertial-frame hovering. Next, we present criteria for stability of body-fixed hovering developed in a previous paper. ${ }^{5}$ This is followed by a discussion of the numerical simulation tool we have developed to aid our study. We then compare results of numerical simulation for two different implementations of body-fixed hovering with the predictions of the analytical stability criteria. Next, we present analytical and numerical analysis of hovering in the inertial frame. Finally, we perform a case study of both hovering approaches for Asteroid (25143) Itokawa (formerly 1998 SF36), the target of the Japan Aerospace Exploration Agency (JAXA) Hayabusa (formerly MUSES-C) mission.,

\section{Problem Formulation}

In this analysis, we model the spacecraft dynamics near a small body using a two-body gravitational model in which the spacecraft has negligible mass. The equations of motion for this system in the uniformly rotating, small-body-fixed Cartesian coordinate frame with origin at the body's center of mass are

$$
\begin{aligned}
\ddot{x}-2 \omega \dot{y} & =\frac{\partial V}{\partial x}+T_{x}+\omega^{2} x \\
\ddot{y}+2 \omega \dot{x} & =\frac{\partial V}{\partial y}+T_{y}+\omega^{2} y \\
\ddot{z} & =\frac{\partial V}{\partial z}+T_{z}
\end{aligned}
$$


where $\omega$ represents the rotation rate of the small body, $V$ is its gravitational potential, and $T_{x}, T_{y}$, and $T_{z}$ represent the component of the spacecraft thrust in each respective direction. It is assumed that the small body has constant density and is rotating uniformly about a fixed axis $(\hat{z})$ corresponding to its maximum moment of inertia. We further orient our coordinate frame by aligning $\hat{\boldsymbol{x}}$ and $\hat{\boldsymbol{y}}$ with the principal axes associated with the body's minimum and intermediate moments, respectively. A position in this space, $[x, y, z]^{T}$, is denoted by the vector $\boldsymbol{r}$. The only forces present in this system are gravitational forces, inertial forces, and forces from the spacecraft's propulsion system. In this paper, we ignore third-body forces of smaller magnitude, such as solar radiation pressure and solar gravity. These additional perturbations will be considered in future work.

We investigate two solutions to these equations. The first solution, which we call body-fixed hovering, creates a fixed equilibrium point in the body-fixed frame at the desired position, or "hovering point," by enabling thrust. Under this control, the spacecraft's position remains fixed relative to the rotating small-body when placed at the nominal hovering point, $\boldsymbol{r}_{0}=\left[x_{0}, y_{0}, z_{0}\right]^{T}$. Enabling thrust is defined as

$$
\boldsymbol{T}=\left[-\left.\frac{\partial V}{\partial x}\right|_{0}-\omega^{2} x_{0},-\left.\frac{\partial V}{\partial y}\right|_{0}-\omega^{2} y_{0},-\left.\frac{\partial V}{\partial z}\right|_{0} ^{T}\right.
$$

The second solution to Eqs. (1-3) that we study is a retrograde, constant-latitude, circular orbit whose orbital period is equal to the small body's rotation period. This solution corresponds to a spacecraft hovering in the inertial reference frame, where the spacecraft position remains fixed in inertial space while the small body rotates beneath it. In the body-fixed frame, this solution is specified as

$\boldsymbol{r}_{0 I}(t)=\left\|\boldsymbol{r}_{0 I}(0)\right\|\left[\cos \lambda_{0} \cos \left(\omega t+\theta_{0}\right), \cos \lambda_{0} \sin \left(\omega t+\theta_{0}\right), \sin \lambda_{0}\right]^{T}$

where $\lambda_{0}$ and $\theta_{0}$ are the initial latitude and longitude (measured from the positive $x$ axis), respectively. For inertial hovering, the necessary thrust vector is defined as that necessary to make this circular orbit a solution to the equations of motion:

$$
\boldsymbol{T}(t)=\left[-\left.\frac{\partial V}{\partial x}\right|_{r_{0 I}(t)},-\left.\frac{\partial V}{\partial y}\right|_{r_{0 I}(t)},-\left.\frac{\partial V}{\partial z}\right|_{r_{0 I}(t)}\right]^{T}
$$

\section{Previous Work}

Sawai et al. ${ }^{5}$ developed a set of criteria analytically that characterize the stability of a hovering trajectory in the body-fixed frame. Through their analysis of the problem, they determined that the Hessian matrix of the gravitational potential at $\boldsymbol{r}_{0}$ determines the stability of hovering in the body-fixed frame subject to their idealized controller:

$$
\left.\frac{\partial^{2} V}{\partial \boldsymbol{r}^{2}}\right|_{0}=\left.\left[\begin{array}{ccc}
\frac{\partial^{2} V}{\partial x^{2}} & \frac{\partial^{2} V}{\partial x \partial y} & \frac{\partial^{2} V}{\partial x \partial z} \\
\frac{\partial^{2} V}{\partial x \partial y} & \frac{\partial^{2} V}{\partial y^{2}} & \frac{\partial^{2} V}{\partial y \partial z} \\
\frac{\partial^{2} V}{\partial x \partial z} & \frac{\partial^{2} V}{\partial y \partial z} & \frac{\partial^{2} V}{\partial z^{2}}
\end{array}\right]\right|_{0}
$$

The conditions for stability they developed are

$$
\begin{aligned}
& 3 \omega^{2} v_{3 z}^{2}+\omega^{2}-\left(\alpha_{1}+\omega^{2}\right)-\left(\alpha_{2}+\omega^{2}\right) \geq 0 \\
& \left(\alpha_{1}+\omega^{2}\right)\left(\alpha_{2}+\omega^{2}\right)-\omega^{2} v_{1 z}^{2}\left(\alpha_{2}+\omega^{2}\right)-\omega^{2} v_{2 z}^{2}\left(\alpha_{1}+\omega^{2}\right) \geq 0 \\
& \left(\alpha_{1}-\alpha_{2}-\omega^{2}\right)^{2}+3 \omega^{4} v_{3 z}^{2}\left(v_{3 z}^{2}+2\right) \\
& \quad-8 \omega^{2} v_{3 z}^{2}\left[\left(\alpha_{1}+\omega^{2}\right)+\left(\alpha_{2}+\omega^{2}\right)\right]>0
\end{aligned}
$$

where $\alpha_{1}, \alpha_{2}, \alpha_{3}$ are the three eigenvalues of the Hessian matrix at $\boldsymbol{r}_{0}$ and $\boldsymbol{v}_{1}, \boldsymbol{v}_{2}, \boldsymbol{v}_{3}$ are the corresponding eigenvectors (subscripts $x, y$, and $z$ refer to the components of these eigenvectors). The eigenvectors and eigenvalues are arranged so that the third set refers to the eigenvector "nearly" aligned with the gravitational attraction direction. Of the remaining two, the eigenvector/eigenvalue pair with the larger eigenvalue is the first set.

These stability criteria were developed by linearizing the equations of motion about $\boldsymbol{r}_{0}$ and assuming one-dimensional, infinitely tight control of motion along $\boldsymbol{v}_{3}$, such that $\dot{\boldsymbol{r}}(t)^{T} \boldsymbol{v}_{3} \equiv 0$. These three criteria define a theoretical region of stability for body-fixed hovering above any arbitrary-shaped body. This region roughly corresponds to the locus of initial hovering points inside the body's resonance radius. The resonance radius $r_{r}$ is defined as the distance from the body's rotation axis in the equatorial plane at which, for a spherical body with the same gravitational parameter $\mu$, the centrifugal force is equal and opposite to the gravitational attraction:

$$
r_{r}=\left(\mu / \omega^{2}\right)^{\frac{1}{3}}
$$

Illustrations of the region defined by these sufficiency criteria for various small-bodies can be seen in Ref. 5 .

The assumption of infinitely tight control is not realistic, as it is impossible for an actual spacecraft's control system to implement. However, similar control can be implemented by use of a dead-band thrust control on altitude, in which the spacecraft's movement is confined to a finite region around a target altitude. Allowing motion across the region defined by such a dead-band may have significant effects on system dynamics. Thus, an analysis using the full nonlinear equations of motion is necessary to validate these results. We further the previous work by numerically simulating the nonlinear equations of motion to determine the stability of hovering under realistic control constraints. We compare and contrast these results with the previous findings to determine in which regions the analytical stability criteria produce valid results.

\section{Numerical Simulation Tool}

Our numerical simulation package was developed to aid us in the analysis of spacecraft trajectories near arbitrary-shaped rotating bodies. The basic function of the code is to integrate the body-fixed equations of motion [Eqs. (1-3)] for a spacecraft in an arbitrary potential field subject to a chosen control law. The code for this simulation was written using Matlab and Simulink software. The equations of motion are integrated using Matlab's "ode45" function (Dormand-Prince method) with a relative tolerance of $10^{-6}(\mathrm{~m})$ and an absolute tolerance of $10^{-8}(\mathrm{~m})$.

The simulation was developed to work with two different asteroid shape and gravity models: the triaxial ellipsoid and the polyhedron. Triaxial ellipsoids are often good approximations of real small-body shapes. Simulations using this shape can help us to understand fundamental phenomena associated with this system that would be difficult to decouple from other effects in the truly arbitrary-shaped case. More complex geometries can be specified to the simulation as $n$-faced polyhedra. Polyhedra can model a much wider range of shapes than ellipsoids, allowing depressions, ridges, cliffs, caverns, and holes on the body. Polyhedron models currently exist for a number of real asteroids. This shape model can be very accurate, with resolution increasing with the number of faces used. Assuming the small body has constant density, previous works exist that exactly define the potential around ellipsoidal ${ }^{8}$ and polyhedral ${ }^{9}$ shapes. The methods described in these papers are implemented in our simulation to calculate gravitational potential, attraction, the Hessian, and the Laplacian.

To ensure that the simulation works as intended, we have run a series of tests with known results and compared the simulation output to the expected answer. First, we confirmed that the spacecraft's angular momentum and energy above a spherical body were conserved throughout the simulation if no thrust was applied. Our next test was to verify that the simulation was computing the correct body forces on the spacecraft. This was done by analytically solving for the forces on the spacecraft at a given point and then adding open-loop 
thrust of equal magnitude and opposite direction to the spacecraft. If the simulation is computing the forces on the spacecraft correctly, this open-loop thrust should cancel those forces and the spacecraft should not move. The simulation was found to correctly produce this result. Our final verification test was done to ensure that the controllers built into the simulator were working as intended. This can be determined by examining the various simulation outputs. Thrust should be enabled in the proper direction whenever the criteria for activation of control are met. We performed this test for many different initial conditions and found the controllers to work as expected.

\section{Stability of Body-Fixed Hovering}

We have investigated the stability of body-fixed hovering subject to two different implementations of the nominal thrust solution [Eq. (4)]. We compare the numerical region of stability obtained with the region defined by the stability criteria [Eqs. (8-10)] derived in the previous work. ${ }^{5}$

Because we are working with numerical data, determining stability characteristics of a trajectory is inherently difficult. With finite-time simulation data, it is impossible to verify true stability or instability. Here, we will be using a modified form of Lyapunov stability suitable to evaluate our trajectories. Beginning with a perturbation from equilibrium smaller than a given magnitude, we quantify stability by the size of the region centered at the initial hovering point that contains the entire trajectory for a given simulation time. We will define instability not as unbounded motion but as motion that extends beyond a specified region during the simulation time. Because of potentially long time constants of instability, this analysis seeks only to verify stability for a fixed duration in the regions predicted by the analytical stability criteria and not to verify instability in the complementary space.

\section{Numerical Simulations of Hovering with Gravitational Direction Thrusting and Sensing with Open Loop Control}

The first body-fixed hovering controller we evaluated is a slightly modified version of the realistic hovering controller suggested in Ref. 5. This controller, which we call the gravitational direction thrusting and sensing with open loop (GDTS w/OL) hovering controller, is a combination of open-loop control thrust to eliminate the spacecraft's nominal acceleration and dead-band control on altitude measured along $\boldsymbol{v}_{3}$. The dead-band controller is defined by a target altitude $h_{0}$ and a tolerance factor $\delta$. If the spacecraft's altitude is outside the "band" defined by $h_{0} \pm \delta$, thrust is enabled in order to return the spacecraft into the dead-band. A spacecraft with an internal model of the small body can compute the Hessian matrix of the potential and determine $\boldsymbol{v}_{3}$. In Ref. 5 , it is suggested that the open-loop control need only cancel the centrifugal force on the spacecraft. However, we have chosen to use the open-loop control to null the full nominal acceleration on the spacecraft, as proposed in Ref. 4, because we have found this type of control to confine the spacecraft's range of motion more tightly. The thrust vector $\boldsymbol{T}$ for the GTDS w/OL controller is

$$
\boldsymbol{T}=\boldsymbol{T}_{o}+\boldsymbol{T}_{\mathrm{db}}
$$

where

$$
\begin{aligned}
& \boldsymbol{T}_{o}=\left[-\left.\frac{\partial V}{\partial x}\right|_{0}-\omega^{2} x_{0},-\left.\frac{\partial V}{\partial y}\right|_{0}-\omega^{2} y_{0},-\left.\frac{\partial V}{\partial z}\right|_{0}\right]^{T} \\
& \boldsymbol{T}_{\mathrm{db}}= \begin{cases}T_{m} \boldsymbol{v}_{3}, & \text { if } \quad\left(h_{0}-h\right)>\delta \\
-T_{m} \boldsymbol{v}_{3}, & \text { if } \quad\left(h_{0}-h\right)<-\delta \\
0, & \text { otherwise }\end{cases}
\end{aligned}
$$

where $T_{m}$ is the constant magnitude of the dead-band thruster and $h$ is the spacecraft altitude. The vector $v_{3}$ should be chosen to point away from the body. This controller mimics the ideal controller used to derive the conditions for stability of body-fixed hovering [Eqs. (8-10)] in the previous work. In that ideal case, the dead-band tolerance factor $\delta$ for this controller is zero. Therefore, a comparison of the performance of this controller with the analytical stability conditions is a direct test of the validity of the idealized result for a spacecraft subject to realistic control.

Using an ellipsoidal small-body shape model, we ran simulations using the GDTS w/OL controller for random initial positions in the body's three symmetric planes $(X Y, X Z$, and $Y Z)$. The ellipsoid used in these simulations was $15 \times 7 \times 6 \mathrm{~km}$, a rough approximation of the size of Asteroid (433) Eros, which was visited during NASA's Near Earth Asteroid Rendezvous (NEAR) mission. A bulk density of $3 \mathrm{~g} / \mathrm{cm}^{3}$ and a 10-h rotation period were used, which are not consistent those of with (433) Eros but were chosen to move the resonance radius further from the body for clarity. (With Eros's true rotation period of $5.27 \mathrm{~h}$ and bulk density of $2.4 \mathrm{~g} / \mathrm{cm}^{3}$, the resonance radius is roughly $15.7 \mathrm{~km}$, barely beyond the long end of the body.) The dead-band tolerance factor $\delta$ was $10 \mathrm{~m}$. For each initial spacecraft position, the simulation was run 10 times with different velocity error each time. Each Cartesian component of velocity error was chosen randomly from a uniform distribution between -1 and $1 \mathrm{~cm} / \mathrm{s}$.

Data were collected from independent simulations of two different durations, 20,000 and 50,000 s (roughly 5.5 and $13.9 \mathrm{~h}$, respectively). These relatively short simulation times (on the order of one rotation period) are justified by noting that a spacecraft operating in the body-fixed frame very near the surface would have little reason to remain in one position for long periods of time. This type of maneuver would likely be used during a descent or a translation across the surface, whereas longer term hovering station-keeping maneuvers would more likely be carried out in the inertial frame.

Our simulations show that in the $X Z$ and $Y Z$ planes the analytical criteria for stability were valid. There were no regions of numerically unstable motion that encroached upon the predicted region of stability. We found that numerical stability extended to some regions outside the area defined by the stability criteria in these planes but these trajectories are not necessarily stable in the long term and, therefore, do not suggest error in the stability conditions.

Figures 1 and 2 present results from simulations in the body's $X Y$ (equatorial) plane for durations of 20,000 and 50,000 s, respectively. In these figures, each data point represents the average size over 10 trials of the smallest solid angle such that the entire trajectory is contained within it. For this analysis, we will consider averages of less than $0.4 \mathrm{deg}$ to be stable. The region inside the bold line is the locus of initial positions satisfying the analytical stability criteria. Note that for an ellipsoidal shape model, the equations of motion exhibit a longitudinal symmetry; that is, results are the same for any two points $180^{\circ}$ longitude apart.

We can see in these figures that the stability criteria do not hold in the $X Y$ plane subject to the GDTS w/OL body-fixed hovering controller. Here, instabilities arise above the body's leading edges in regions satisfying the stability conditions. The leading edges are defined as the two quadrants of longitude on the body's surface that extend from the tip of the largest semimajor axis of the ellipsoid to the intermediate semimajor axis in the direction of $\hat{\boldsymbol{z}} \times \hat{\boldsymbol{x}}$. As the simulation duration increases from 20,000 to $50,000 \mathrm{~s}$, we can see that the area of instability encroaches further upon the region satisfying the stability criteria. On the other hand, the region of predicted stability above the body's trailing edge is unaffected and remains stable. We also find the region of stability off the trailing edge to be significantly expanded for shorter duration (20,000 s) hovering.

\section{Analysis of GDTS w/OL Controller Results}

We attribute the discrepancy between the ideal region of stability and our numerical findings to the Coriolis accelerations introduced by relaxing the infinitely tight control in the gravitational direction assumed in the analytical work. An interaction between the dead-band orientation, the control direction, and the Coriolis accelerations causes the degradation of stability above the ellipsoid's leading edges and the improvement in stability above the trailing edge that are seen in our simulations.

The orientation of the dead-band region for hovering in the $X Y$ plane is shown in Fig. 3. We define a coordinate system for the deadband dynamics consisting of the normal vector to the dead-band 


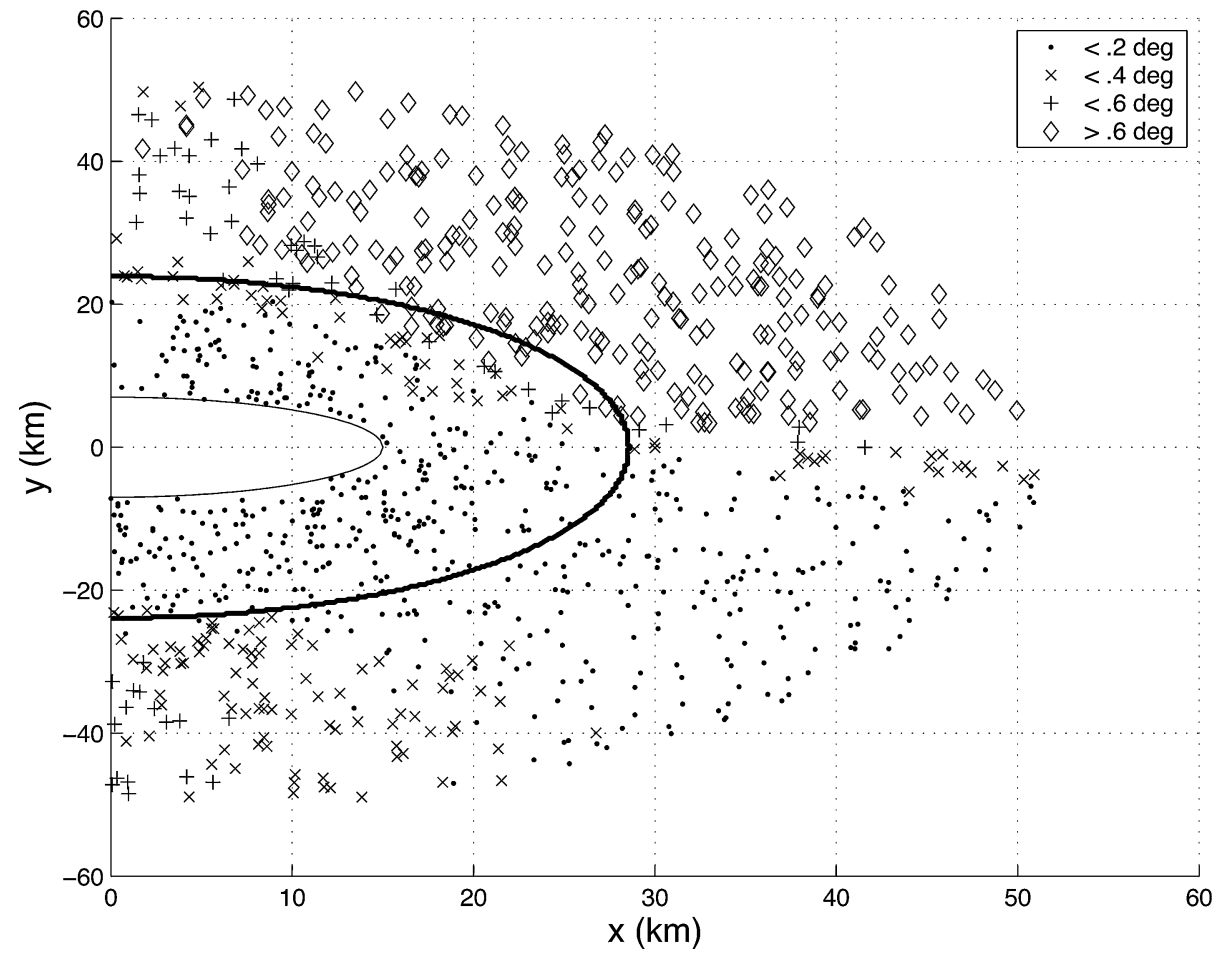

Fig. 1 Stability results for GDTS w/OL body-fixed hovering controller. Angular deviation 20,000 s (XY plane).

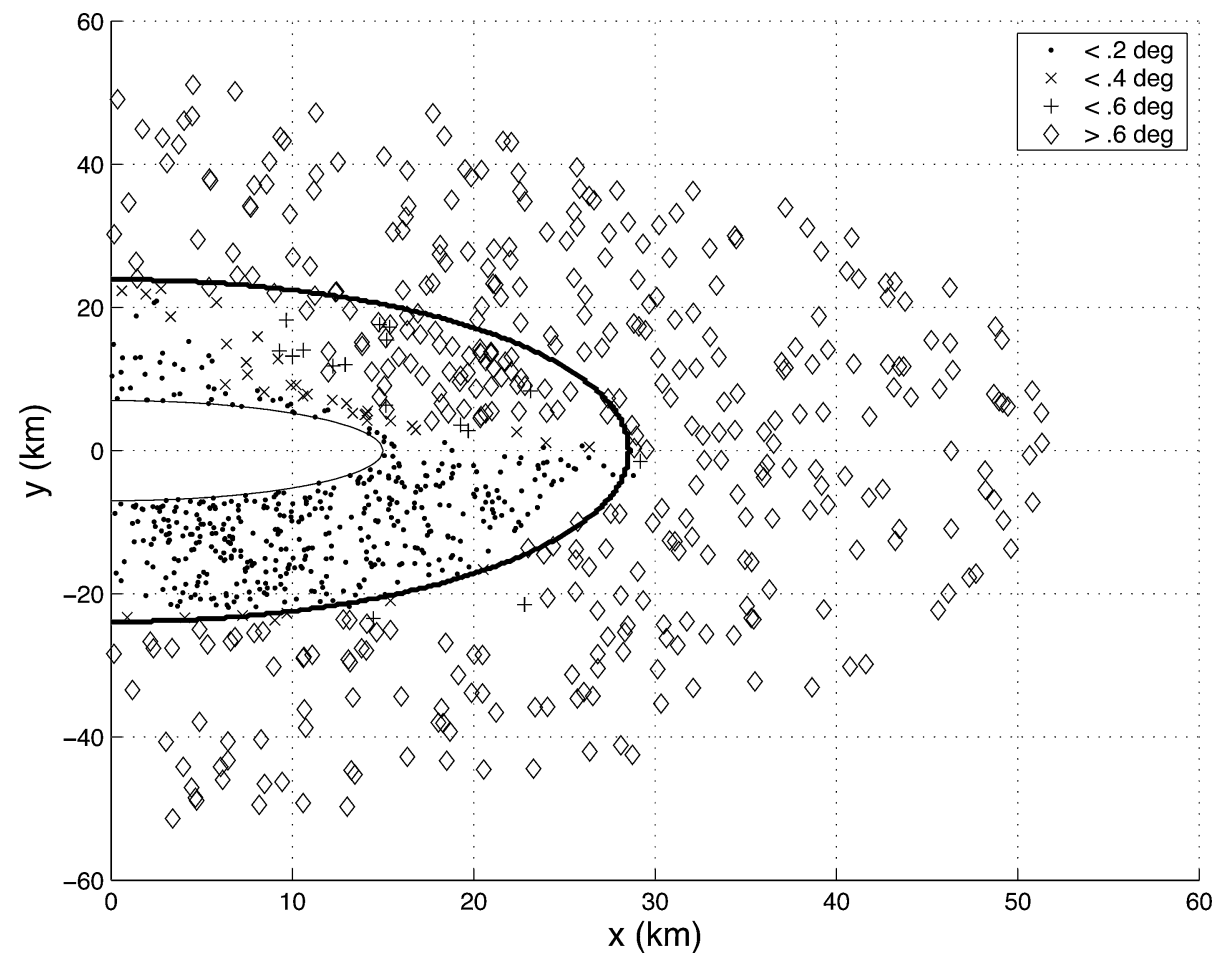

Fig. 2 Stability results for GDTS w/OL body-fixed hovering controller. Angular deviation 50,000 s (XY plane).

boundary, $\boldsymbol{n}_{\mathrm{db}}$, the unit vector along the rotation axis, $\hat{z}$, and the vector transverse to the dead band, $\boldsymbol{t}_{\mathrm{db}}=\hat{\boldsymbol{z}} \times \boldsymbol{n}_{\mathrm{db}}$. The surface normal where the altitude-sensing-direction vector $\boldsymbol{v}_{s}$ intersects the body's surface defines $\boldsymbol{n}_{\mathrm{db}}$, the local orientation of the dead band. This direction, in general, is not aligned with the control direction $\boldsymbol{v}_{c}$. Therefore, when control thrust is applied, it will have some component along $\boldsymbol{t}_{\mathrm{db}}$. This transverse thrust component will be equal and opposite at the two dead-band boundaries. If the spacecraft hits both boundaries an equal number of times, this transverse acceleration will have no net effect on the spacecraft's motion along the dead band. However, because of Coriolis forces, which effectively rotate the spacecraft's velocity vector, this may not be the case. This rotation, coupled with the control thrust component along the dead band, can either cause the spacecraft to repeatedly hit one boundary of the dead band or encourage it to bounce back and forth between the boundaries.

Figure $4 \mathrm{a}$ illustrates the dynamics above the leading edge. Above an ellipsoid's leading edge subject to the GDTS w/OL hovering controller, the relative orientation of $\boldsymbol{n}_{\mathrm{db}}$ and $\boldsymbol{v}_{c}$ is such that at the minimum altitude boundary of the dead-band, a component of the control thrust is applied in the negative $\boldsymbol{t}_{\mathrm{db}}$ direction and at the maximum 


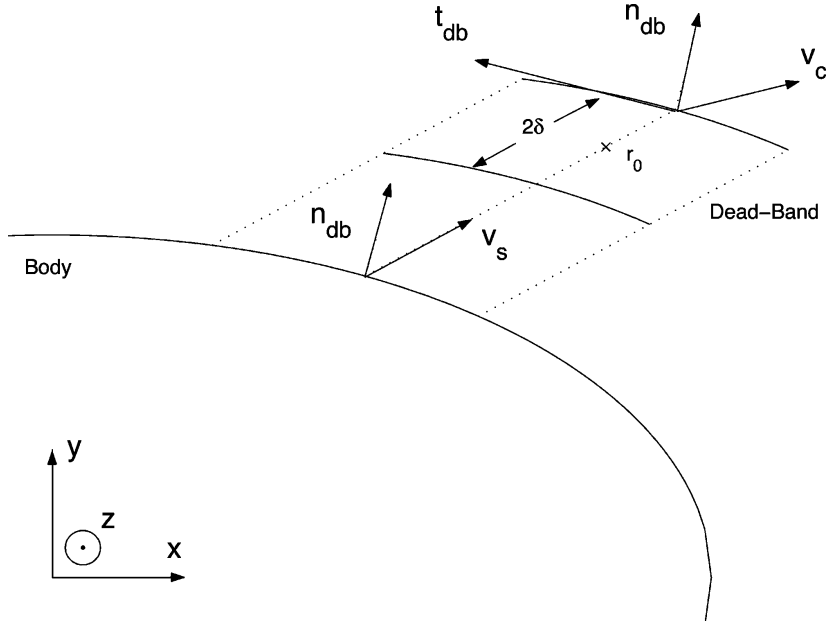

Fig. 3 Dead-band orientation diagram.

altitude boundary, thrust is applied in the positive $t_{\mathrm{db}}$ direction. The Coriolis force acting on the spacecraft, $F_{c}=-2 \omega(\hat{z} \times \dot{\boldsymbol{r}})$, effectively rotates the spacecraft's velocity vector clockwise in the plane defined by $\boldsymbol{n}_{\mathrm{db}}$ and $\boldsymbol{t}_{\mathrm{db}}$. When the spacecraft reaches a boundary, the component of control thrust along $\boldsymbol{t}_{\mathrm{db}}$ will effectively rotate the return velocity vector clockwise from the direction it would have had if there had been no thrust component along $\boldsymbol{t}_{\mathrm{db}}$. As the spacecraft moves toward its next boundary crossing, Coriolis forces will rotate the velocity in the same clockwise direction. Once transients due to initial velocity errors wear off, the combination of these two effects will cause the spacecraft to hit the same boundary on successive occasions until the nonlinear effects of gravity and changes in dead-band orientation eventually turn it around. This successive "bouncing" motion is linearly unstable and leads to large oscillations from the nominal hovering point. Examination of numerical simulations shows that this bouncing movement always takes over in the steady state for hovering above the leading edge of an ellipsoid subject to the GDTS w/OL controller.

Figure $4 \mathrm{~b}$ illustrates the dynamics above the trailing edge. Above the body's trailing edge, the relative orientation of $\boldsymbol{n}_{\mathrm{db}}$ and $\boldsymbol{v}_{c}$ is opposite, such that at the minimum altitude boundary, a component of the control thrust is applied in the positive $t_{\mathrm{db}}$ direction and at the maximum altitude boundary, thrust is applied in the negative $\boldsymbol{t}_{\mathrm{db}}$ direction. As opposed to above the leading edge, the thrust component along $t_{\mathrm{db}}$ in this configuration causes the velocity vector reflected off the dead-band boundary to be rotated counterclockwise from where it would have been if there were no transverse thrust component. In this case, the clockwise rotation of the velocity vector caused by the Coriolis force will counter the effect of the control thrust, encouraging the spacecraft to move toward the other dead-band boundary. Again, in the steady state, this motion always takes over above an ellipsoid's trailing edge, causing a "chattering" effect, where the spacecraft never hits the same boundary on successive occasions. This chattering has a stabilizing effect that keeps the spacecraft close to the initial hovering point.

The analysis here is done in the equatorial plane for the sake of simplicity, but this effect occurs with weakening strength all the way to the body's $X Z$ and $Y Z$ planes. In these planes, thrust does not directly induce motion that causes disruptive Coriolis accelerations. This is why our numerical results in the $X Z$ and $Y Z$ planes agree well with the analytical predictions, in whose derivation Coriolis forces essentially do not exist. The strength of this Coriolis effect above either the leading or trailing edge is determined by $\omega, \delta$, and the angle between $\boldsymbol{n}_{\mathrm{db}}$ and $\boldsymbol{v}_{c}$. If we define this angle as $\theta$, the less negative $\theta$ is (measured from $\boldsymbol{n}_{\mathrm{db}}$ and positive for orientations such that $\boldsymbol{v}_{c}^{T} \boldsymbol{t}_{\mathrm{db}}>0$ ), the weaker the destabilizing effect of control thrust will be above the leading edge, whereas the more positive $\theta$ is, the stronger the focusing effect will be above the trailing edge. If we use a symmetric, universal method of choosing control direction, such as gravitational direction or initial acceleration, we can postulate that some optimal control law exists that will adequately focus the leading edge dynamics, while not weakening the focusing effect off the trailing edge too much. This optimal control direction would be a function of small-body parameters as well as the hovering duration. Ideally, a spacecraft would use a method of choosing control direction above the leading and trailing edges such that the angle $\theta$ is always positive. We have been able to show through numerical simulation that shifting the control direction above the leading edge so that $\theta$ is positive does indeed produce the desired stabilizing effect. It should be noted that $\theta$ should never approach $\pm 90 \mathrm{deg}$ because there would then be no thrust in the direction normal to the dead-band to maintain the spacecraft's altitude.

\section{Numerical Simulations of Hovering with Initial Acceleration Thrusting and Normal Sensing Control}

We have also performed numerical simulations using another implementation of the body-fixed hovering solution that we call initial acceleration thrusting and normal sensing (IATNS) hovering control. We still use a dead-band controller based on altimeter readings, but we eliminate the open-loop thrust that cancels the spacecraft's nominal accelerations. For the IATNS controller, we also change the direction of the dead-band control thrust to be aligned with the nominal acceleration vector to more directly counter the natural motion of the spacecraft. The direction of altitude measurement is also changed from along $\boldsymbol{v}_{3}$ to measure altitude in the direction normal to the surface at the hovering point. This is the most robust direction in which to measure altitude because the component of altitude that changes with the surface topology is minimized for small deviations in spacecraft position.

The IATNS controller offers advantages over the GDTS w/OL controller. The elimination of the open-loop thrust will result in fuel savings because with an open-loop thrust, any inaccuracies in its application will result in a constant push toward one side of the dead band that must be negated with thrust from the closed-loop controller, effectively causing the control to work against itself. Also, removing the open loop causes the spacecraft's motion to proceed very closely along the initial acceleration direction from the initial hovering point. This lessens the deviation from the initial hovering point due to transient effects caused by initial velocity errors because the spacecraft will more quickly move into steady-state motion in the dead band. Sensing altitude in the direction normal to the surface at the initial hovering point keeps the dead-band orientation vector $\boldsymbol{n}_{\mathrm{db}}$ closer to the control direction $\boldsymbol{v}_{c}$, which our analysis suggests should improve performance above the body's leading edge. This controller may also offer advantages in a surface-descent scenario, as there will exist some angle between the thruster plume and the surface normal. This angle could help avoid contamination of the surface regolith by the thrusters in a sample collecting mission and allow descent imaging to be less obscured by the thruster plume. The thrust vector for the IATNS controller is

$$
\boldsymbol{T}= \begin{cases}-T_{m} \hat{\boldsymbol{a}}_{0}, & \text { if } \quad h_{0}-h>\delta \\ T_{m} \hat{\boldsymbol{a}}_{0}, & \text { if } \quad h_{0}-h<-\delta \\ 0, & \text { otherwise }\end{cases}
$$

where

$$
\begin{gathered}
\hat{\boldsymbol{a}}_{0}=\left[\left.\frac{\partial V}{\partial x}\right|_{0}+\omega^{2} x_{0},\left.\frac{\partial V}{\partial y}\right|_{0}+\omega^{2} y_{0},\left.\frac{\partial V}{\partial z}\right|_{0}\right]^{T} / \\
\left\|\left[\left.\frac{\partial V}{\partial x}\right|_{0}+\omega^{2} x_{0},\left.\frac{\partial V}{\partial y}\right|_{0}+\omega^{2} y_{0},\left.\frac{\partial V}{\partial z}\right|_{0}\right]^{T}\right\|
\end{gathered}
$$

With some thought, we expect that the IATNS controller will fail catastrophically near the resonance radius of the body. As the spacecraft hovering point approaches the resonance radius, the initial acceleration vector $\hat{\boldsymbol{a}}_{0}$ will turn $180 \mathrm{deg}$ as the nominal acceleration transitions from pointing toward the body to away. Therefore, this 


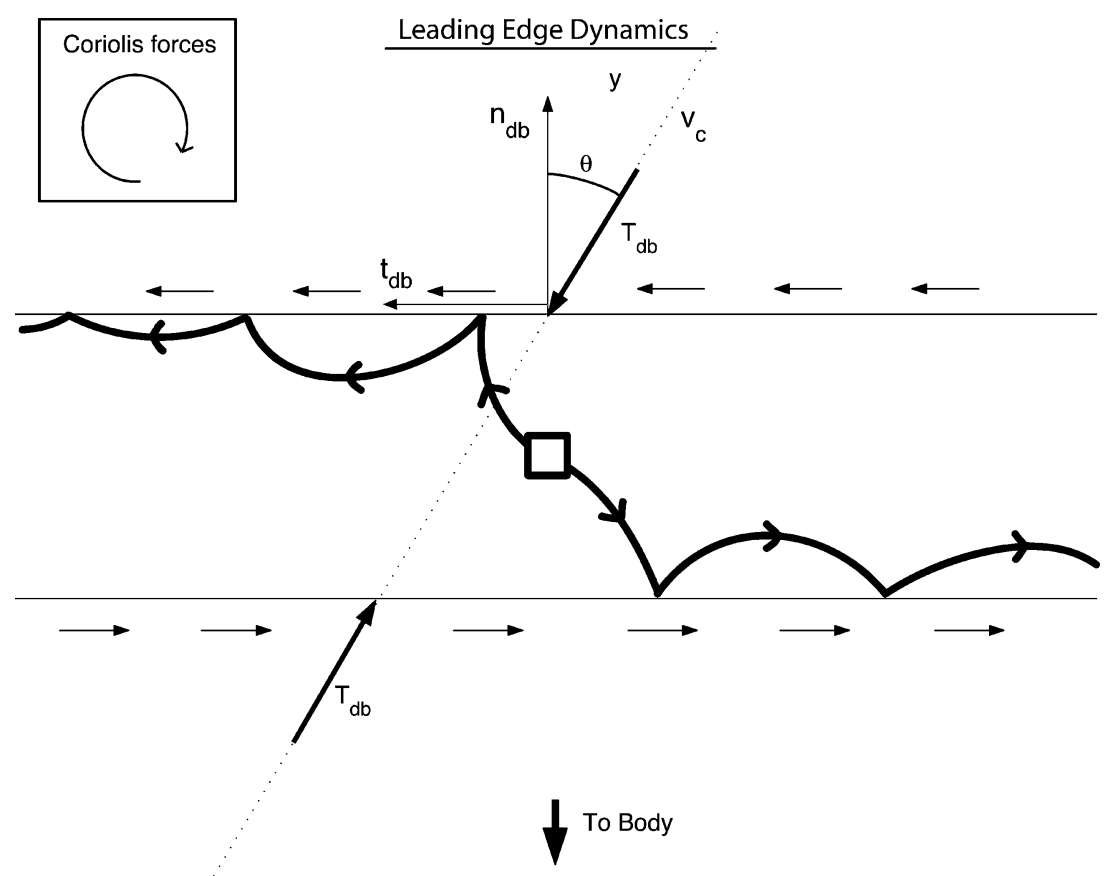

a)

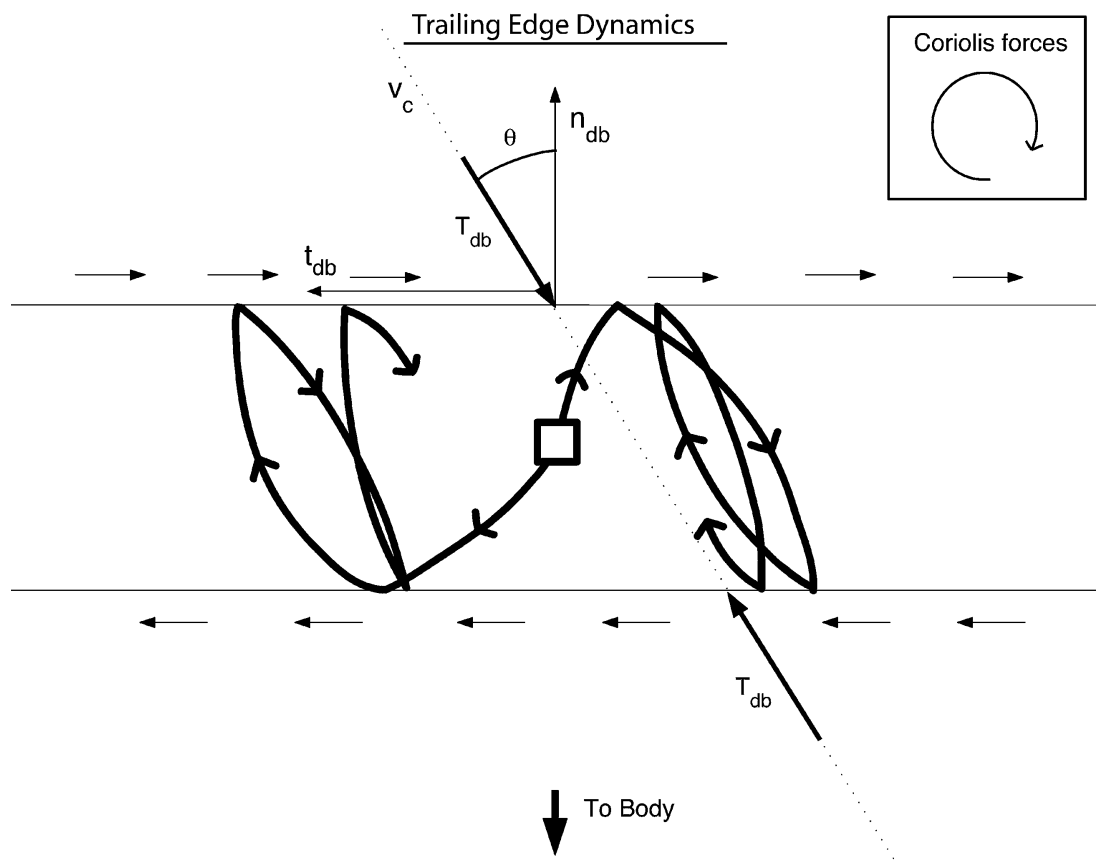

b)

Fig. 4 Dead-band dynamics above an ellipsoid under GDTS w/OL control: a) leading and b) trailing edges.

controller will be limited to use inside the body's resonance radius or near the rotation axis.

Using the same ellipsoidal small-body and system parameters used in our simulations for the GDTS w/OL controller, we have simulated spacecraft trajectories subject to the IATNS controller in the $X Y, X Z$, and $Y Z$ planes. Again, we ran 10 different simulations with random velocity errors at each initial hovering point.

We found the IATNS body-fixed hovering controller to work very well at altitudes inside the resonance radius. As expected, the controller quickly becomes less stable as the initial position nears the area where the initial acceleration rotates away from the body. In all three planes, initial positions inside this threshold were found to be stable. For comparison, Fig. 5 shows the results of simulations in the $X Y$ plane after $50,000 \mathrm{~s}$. In the $X Y$ plane, the analytical stability conditions define the threshold where the initial acceleration vector turns away from the body. The bold line in the figure outlines this region. As we hoped, the IATNS controller reduces the deviations associated with the Coriolis forces discussed in the preceding section above the body's leading edge compared to the GDTS w/OL controller. By reducing the control thrust component along the dead band, the instability related to Coriolis forces above the leading edge is weakened and develops more slowly. In this 50,000-s figure, a slight degradation of stability is just beginning to appear above the leading edge. In the data from the 20,000-s runs, this degradation of stability cannot be seen. Similarly, the stabilizing effect above the trailing edge should be slightly weakened by the realignment of the 


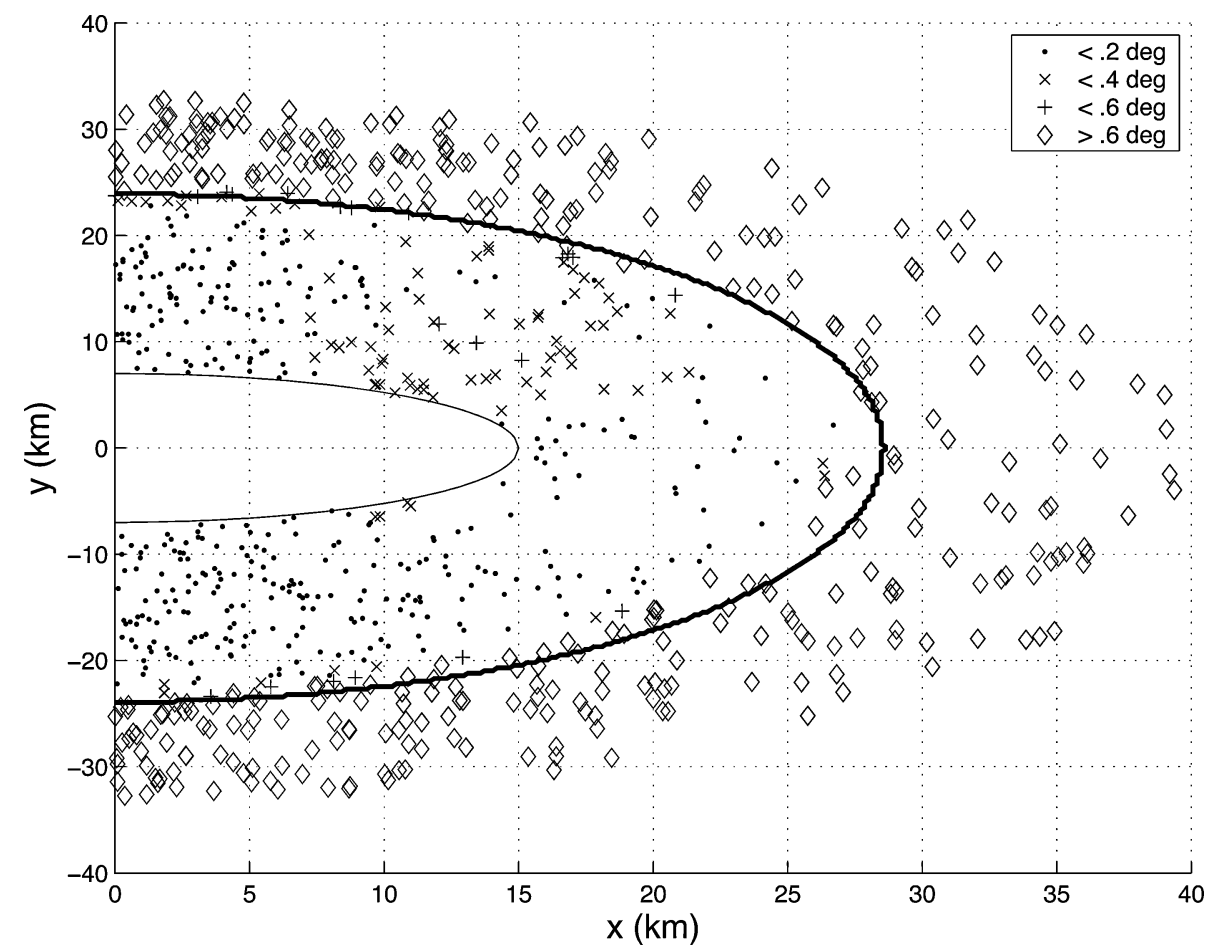

Fig. 5 Stability results for IATNS body-fixed hovering controller. Angular deviation 50,000 s ( $X Y$ plane).

vector $\boldsymbol{n}_{\mathrm{db}}$ for this controller. However, the angle $\theta$ is still negative and therefore a stabilizing effect remains, as demonstrated in our numerical results.

\section{Stability of Inertial Hovering}

We now look at the equations of motion for inertial hovering in the body-fixed frame [Eqs. (1-3) and (5)]. Linearizing about this solution [Eq. (5)], we obtain the perturbation equations

$$
\begin{gathered}
\Delta \ddot{x}-2 \omega \Delta \dot{y}-\omega^{2} \Delta x=\left.\frac{\partial^{2} V}{\partial x^{2}}\right|_{0} \Delta x+\left.\frac{\partial^{2} V}{\partial x \partial y}\right|_{0} \Delta y+\left.\frac{\partial^{2} V}{\partial x \partial z}\right|_{0} \Delta z \\
\Delta \ddot{y}+2 \omega \Delta \dot{x}-\omega^{2} \Delta y=\left.\frac{\partial^{2} V}{\partial x \partial y}\right|_{0} \Delta x+\left.\frac{\partial^{2} V}{\partial y^{2}}\right|_{0} \Delta y+\left.\frac{\partial^{2} V}{\partial y \partial z}\right|_{0} \Delta z \\
\Delta \ddot{z}=\left.\frac{\partial^{2} V}{\partial x \partial z}\right|_{0} \Delta x+\left.\frac{\partial^{2} V}{\partial y \partial z}\right|_{0} \Delta y+\left.\frac{\partial^{2} V}{\partial z^{2}}\right|_{0} ^{\Delta z}
\end{gathered}
$$

This is a periodic, time-varying linear system. We can analyze the stability of this system using Floquet theory, which suggests that the state transition matrix is of the form $\Phi=P(t) \exp (M t)$, where $P(t)$ is a periodic matrix and $M$ is a constant matrix. We can evaluate the stability of the system by examining the eigenvalues of $M$. This is done by evaluating the state transition matrix after one period of motion, that is, when $P$ is the identity matrix. We can numerically calculate the state transition matrix using our simulation package. Further, we note that this is a Hamiltonian system, and thus, the eigenvalues of $M$, or Floquet multipliers, must come in complex conjugate and inverse pairs. This means that for stability, the Floquet multipliers of the system must all lie on the unit circle in the complex plane. Multipliers with magnitude different from unity imply instability.

Without performing any calculations, we expect that the system will have at least two unstable multipliers corresponding to motion in roughly the radial direction. If there are some perturbations in this direction, the gravitational attraction the spacecraft feels will either increase or decrease in the same way that the nominal thrust will be inaccurate, resulting in an unchecked acceleration.

We can validate this insight by looking at the simplified case of inertial hovering over a rotating point mass. Because this potential field is spherically symmetric, it will remain constant throughout the maneuver. The equations of motion in the inertial frame for this system are

$$
\begin{aligned}
& \ddot{x}=\frac{\partial V}{\partial x}+T_{x}=-\frac{\mu}{r^{3}} x+T_{x} \\
& \ddot{y}=\frac{\partial V}{\partial y}+T_{y}=-\frac{\mu}{r^{3}} y+T_{y} \\
& \ddot{z}=\frac{\partial V}{\partial z}+T_{z}=-\frac{\mu}{r^{3}} z+T_{z}
\end{aligned}
$$

where $r=\left(x^{2}+y^{2}+z^{2}\right)^{\frac{1}{2}}$ is the radial distance from the point mass. If we linearize about a nominal position $\left[x_{0}, y_{0}, z_{0}\right]^{T}$ and thrust vector,

$$
\boldsymbol{T}_{0}=\left[-\left.\frac{\partial V}{\partial x}\right|_{0},-\left.\frac{\partial V}{\partial y}\right|_{0},-\left.\frac{\partial V}{\partial z}\right|_{0} ^{T}\right.
$$

we obtain the following perturbation equations:

$$
\left[\begin{array}{c}
\ddot{\Delta} x \\
\ddot{\Delta y} \\
\ddot{\Delta z}
\end{array}\right]=\left.\mu\left[\begin{array}{ccc}
\frac{3 x^{2}}{r^{5}}-\frac{1}{r^{3}} & \frac{3 x y}{r^{5}} & \frac{3 x z}{r^{5}} \\
\frac{3 x y}{r^{5}} & \frac{3 y^{2}}{r^{5}}-\frac{1}{r^{3}} & \frac{3 y z}{r^{5}} \\
\frac{3 x z}{r^{5}} & \frac{3 y z}{r^{5}} & \frac{3 z^{2}}{r^{5}}-\frac{1}{r^{3}}
\end{array}\right]\right|_{0}\left[\begin{array}{c}
\Delta x \\
\Delta y \\
\Delta z
\end{array}\right]
$$

Because the gravitational field is spherically symmetric, we can assume, without loss of generality, that the initial position is of the 


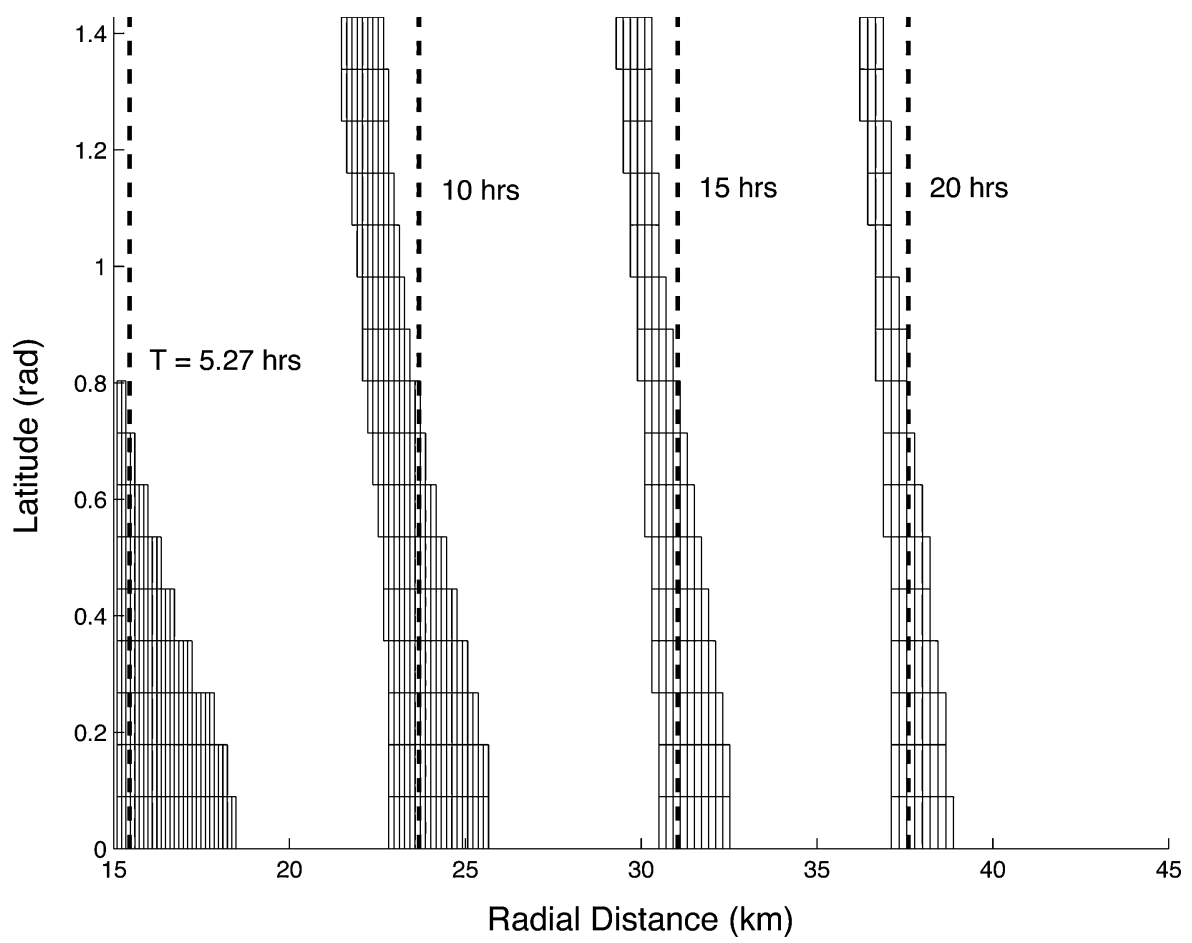

Fig. 6 Regions of instability for inertial hovering above a $15 \times 7 \times 6$ ellipsoid for various rotation rates, radius vs latitude, $T_{E r o s}=5.27 \mathrm{~h}$.

form $\left[x_{0}, 0,0\right]^{T}$. These equations then reduce to

$$
\left[\begin{array}{c}
\ddot{\Delta x} \\
\ddot{\Delta y} \\
\ddot{\Delta z}
\end{array}\right]=\mu\left[\begin{array}{ccc}
\frac{2}{x_{0}^{3}} & 0 & 0 \\
0 & -\frac{1}{x_{0}^{3}} & 0 \\
0 & 0 & -\frac{1}{x_{0}^{3}}
\end{array}\right]\left[\begin{array}{c}
\Delta x \\
\Delta y \\
\Delta z
\end{array}\right]
$$

These are three decoupled, second-order differential equations. The eigenvector along the radial direction is unstable with eigenvalues $\pm \sqrt{ }\left(2 \mu / x_{0}^{3}\right)$ and the two eigenvectors transverse to the radial direction have stable eigenvalues equal to $\pm i \sqrt{ }\left(\mu / x_{0}^{3}\right)$.

Here, a stabilizing controller is necessary for the radial direction, as predicted, and the transverse directions are always stable. The stability of the transverse eigenvalues will change in the more general case, when the potential distribution varies as the body rotates. For this discussion of stability, we will ignore the inherently unstable eigenvalues, assuming existence of a stabilizing controller in the radial direction (similar to the tight control assumed in Ref. 5).

For the ellipsoidal shape case, we have numerically integrated the perturbation equations [Eqs. (17-19)] for one period of motion and determined the eigenvalues of the state transistion matrix. Integrations were again performed using Matlab with a relative tolerance of $10^{-8}(\mathrm{~m})$ and an absolute tolerance of $10^{-11}(\mathrm{~m})$. We found that inertial-frame hovering, subject to a stabilizing controller in the radial direction, is stable at most radial distances and inclinations. The exception to this is a region near the resonance radius that extends around the body, forming a nearly spherical "shell" of instability. The cross-hatched regions in Fig. 6 show the shape of this unstable region, which is axially symmetric about $\hat{z}$ for different rotation rates around an ellipsoidal body measuring $15 \times 7 \times 6 \mathrm{~km}$ with a density of $2.3 \mathrm{~g} / \mathrm{cm}^{3}$. The resonance radius corresponding to each rotation rate is shown as a vertical dashed line.

It can be seen that the region of instability moves with the changing resonance radius [Eq. (11)], a function of rotation rate. As latitude increases from the ellipsoid's equator to its pole, the radius of unstable hovering becomes smaller and the strength of the instability is marginally decreased. The radial distance covered by the instability also decreases as the rotation rate decreases. Figure 7 shows the magnitude of the four transverse eigenvalues (corresponding to the two transverse directions in position space) vs the radius in the equatorial plane for selected rotation rates. Again, the correlation between the primary instability and the resonance radius is clearly shown. This figure also shows that the magnitude of the instability increases for faster rotation rates. We note that the magnitude of the instabilities that exist near the resonance radii is small (generally $<2$ ). Beyond the instability at the body's resonance radius, we have found hovering to be stable in all cases up to the point where our assumptions break down and third-body effects of the sun become an issue. The qualitative properties of this ellipsoidal example are reflective of the other ellipsoidal shapes we have tested.

One may expect there to be instabilities near other harmonics of the rotation rate. Our data have revealed that relatively weak instabilities may exist inside the resonance radius at these secondary resonances. However, as the ratio of hovering radius to resonance radius decreases, the condition number of the state transition matrix that gives us our stability result increases exponentially. The secondary harmonic instabilities we have found for the ellipsoidal body case lie too far inside the resonance radius to be considered numerically accurate. Therefore, the stability of hovering well inside the resonance radius remains unknown. We have found no secondary harmonics outside of the resonance radius, where the result is numerically tractable.

\section{Numerical Support}

The analytical result described above is supported very well by numerical simulation. For these simulations, we used an ellipsoidal shape model with dead-band control on distance from the plane passing through the origin defined by the vectors $\hat{z}$ and $\boldsymbol{r}_{0} \times \hat{z}$ in the inertial frame. The control direction was defined as being constant and in the direction of the initial hovering point.

Because the control direction is constant and the dead-band control confines the motion of the spacecraft to a thin region between two planes, we can imagine that, assuming the thrusters have adequate authority, all inertial hovering trajectories will at least have 


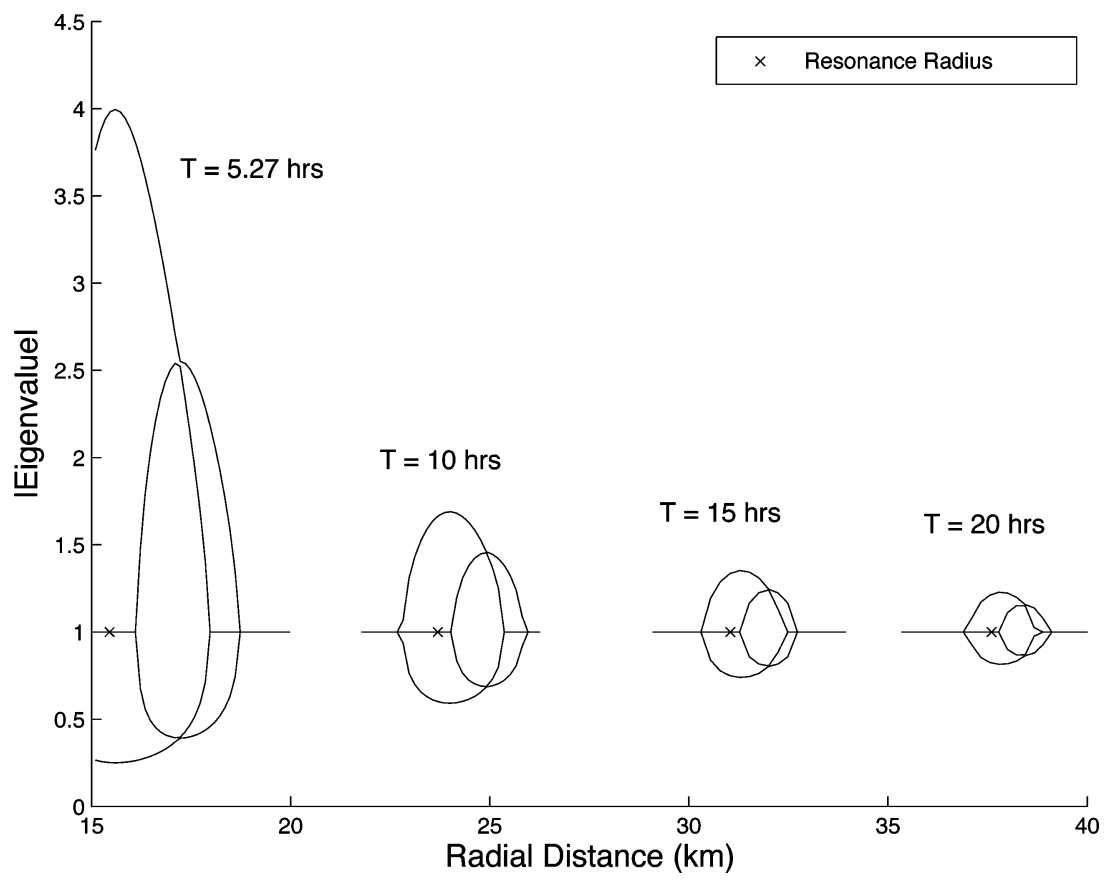

Fig. 7 Magnitude of Floquet multipliers for inertial hovering above a $15 \times 7 \times 6$ ellipsoid for various rotation rates. Latitude $=0^{\circ}$.

bounded time responses. That is, as the spacecraft moves far from the initial point, the gravitational attraction will increasingly pull the spacecraft back toward center. This phenomenon is not apparent in the linearized analysis but is ultimately true in the full nonlinear case. However, oscillations of this magnitude generally would not be acceptable during a mission to a small body. For the purposes of our analysis, we will define instability not as unbounded motion but as growth in the periodic oscillation of the spacecraft about the nominal point. This view is consistent with the linearized stability used in the analytical analysis.

We performed simulations of inertial hovering trajectories over a range of radial distances along the equatorial plane. The primary feature of the previous results, instability near the resonance radius, was clearly shown for trajectories with a target radius near the resonance radius. Numerical simulation also confirmed that the linear analytical stability analysis for hovering outside the resonance radius produced accurate results; that is, inertial hovering is stable at all radii beyond the resonance radius instability. The oscillations in the position of the spacecraft in these regions were seen to be bounded for relatively long simulation times (35 days). In these simulations, we also found that in regions very near the body, oscillations from the nominal point were quite large in magnitude, though bounded in the long term. In general, the bound on the spacecraft's oscillations about the nominal point for stable hovering radii became smaller as radius increased. Recall that our analytical work did not yield insight to the stability of inertial hovering very near the body. Our numerical results have shown inertial hovering to be nonlinearly stable very near the body, but with large (on the order of the small-body size) oscillations in position.

\section{Hovering Above Asteroid Itokawa}

On 9 May 2003, JAXA successfully launched the MUSES-C spacecraft, renamed Hayabusa after launch, toward (25143) Itokawa. ${ }^{10}$ In this mission, the spacecraft will rendezvous with the asteroid, spend some time in inertial hovering, and then descend to the surface to collect regolith samples to be returned to Earth. Because of the applicability of our work to this mission, we now present results for a spacecraft operating near Itokawa subject to our control strategies. We have simulated motion above the 6098-vertex polyhedral shape model of Itokawa developed by Ostro et al., ${ }^{11}$ using their estimates of density $\left(2.5 \mathrm{~g} / \mathrm{cm}^{3}\right)$ and rotation period $(12.132 \mathrm{~h})$.

\section{Body-Fixed Hovering}

To verify that the qualitative results found for the ellipsoidal case apply to more realistic small-body shapes, we have performed simulations of body-fixed hovering above Itokawa subject to the two controllers discussed earlier with a $\delta$ of $5 \mathrm{~m}$. Our approach was to use initial positions at a range of altitudes along various radial lines extending from the center of the body to capture the qualitative stability properties found in the ellipsoidal case. Again, we quantify stability by average maximum angular deviation over 20 runs with different initial velocity errors.

Recall that in the ellipsoidal case, the GDTS w/OL controller [Eq. (12)] agreed well with the analytical stability criteria in the $X Z$ and $Y Z$ planes. In the equatorial plane, there were unstable regions found above the body's leading edge that satisfied the stability criteria and the stable region was extended above the trailing edge for shorter duration hovering. Our analysis has suggested that these leading- and trailing-edge effects are present at all latitudes, weakening as the hovering point moves away from the equator. These results are supported by numerical simulations of GDTS w/OL hovering above Itokawa.

For the radial line extending at $30^{\circ}$ latitude and $45^{\circ}$ longitude (measured from the positive $x$ axis), we found the GDTS w/OL controller to behave in line with the stability criteria. There is a clearly visible increase in the average angular deviation at both 20,000 and 50,000 s when the initial radius moves beyond the region satisfying the stability criteria. As the initial radius approaches the limit of the analytically stable region, a slow increase in average deviation is found, which we attribute to the weakened, out-of-plane, leading edge effect.

Figure 8 shows angular deviation versus radius for GDTS w/OL hovering at $5^{\circ}$ latitude and $45^{\circ}$ longitude, that is, above the leading edge near the equatorial plane. The radii satisfying the analytical stability criteria are indicated with squares at the bottom of the plot. We can see that the average deviation starts to increase somewhat inside the region satisfying the sufficiency criteria for the 20,000-s runs. For the 50,000-s runs, the effect is more dramatic; destabilization occurs well inside the limit of analytical stability and causes severe degradation in performance. These findings support the results of the ellipsoidal analysis.

Simulations for the radial line at $0^{\circ}$ latitude and $-30^{\circ}$ longitude, that is, off the trailing edge, show a region of stability extending well beyond the threshold suggested by the analytical stability criteria after $20,000 \mathrm{~s}$. In the 50,000-s case, the angular deviation 


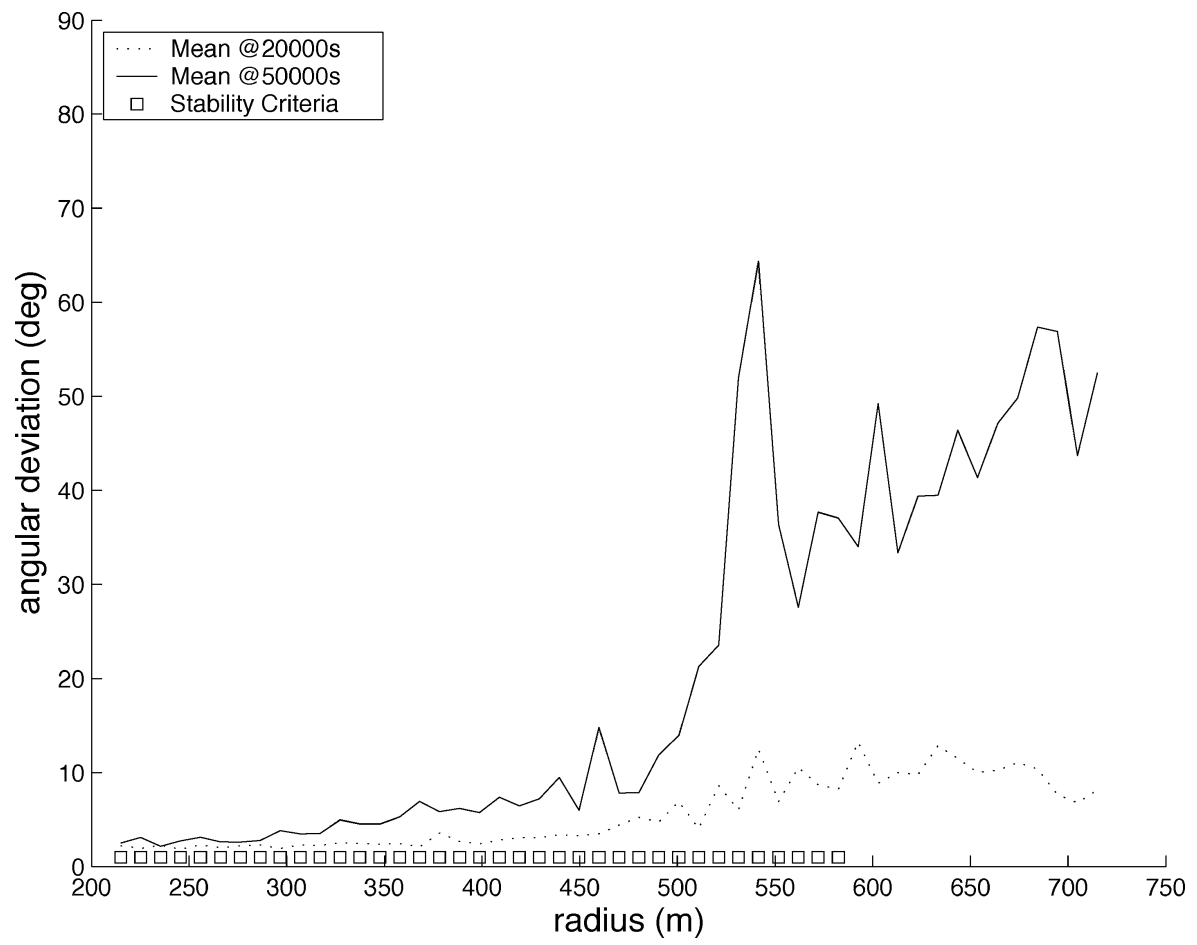

Fig. 8 Stability of body-fixed hovering above Asteroid (25143) Itokawa as a function of radius, GDTS w/OL controller $\left(5^{\circ}\right.$ latitude, $45^{\circ}$ longitude).

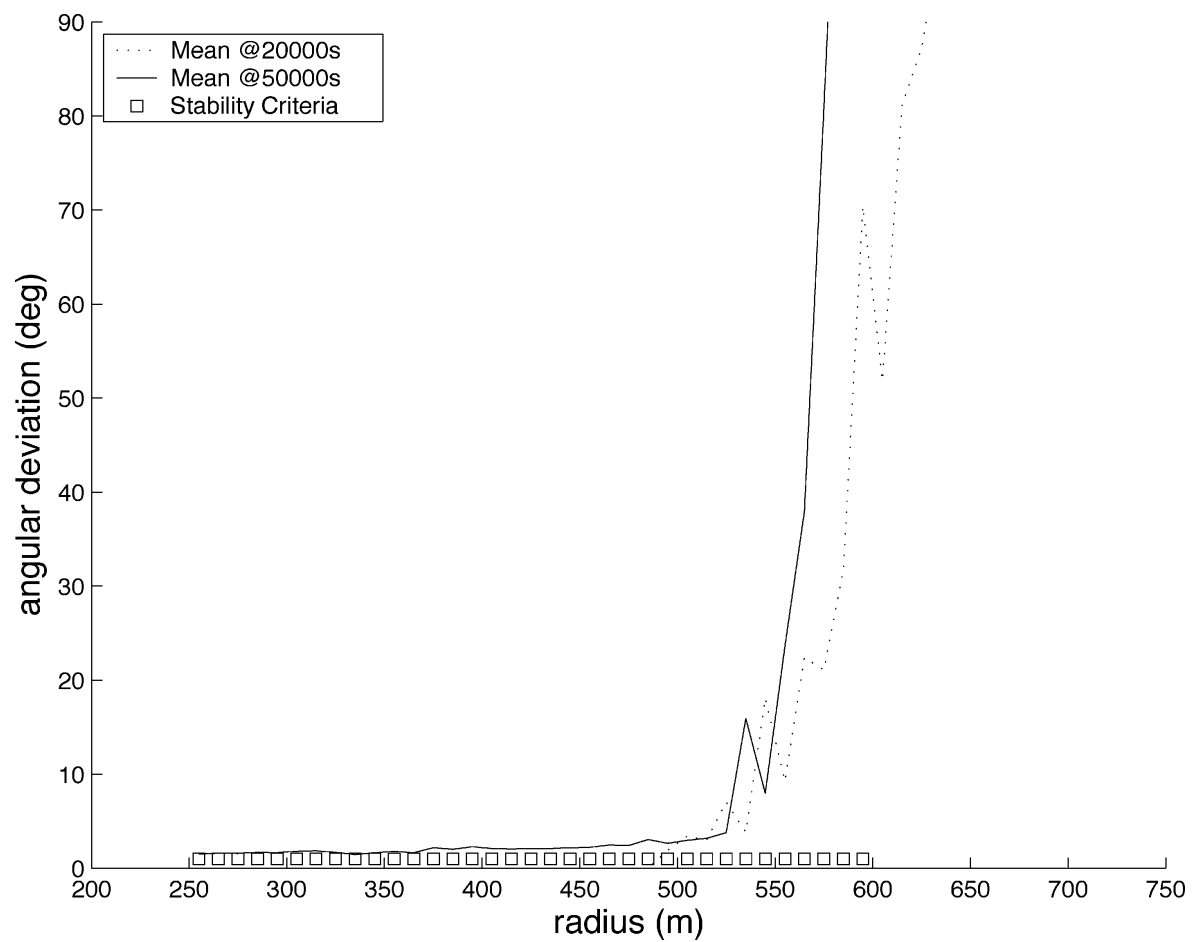

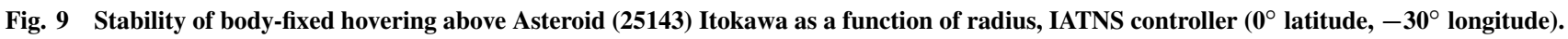

begins to increase just inside the limit of the region of predicted stability. Beyond the analytical stability threshold, deviations are noticably smaller than in the unstable regions of the other two cases.

We also implemented the IATNS controller [Eq. (15)] above Itokawa. Again, we found the results to be in agreement with our findings in the ellipsoidal case. That is, the IATNS controller is consistently stable for 20,000- and 50,000-s simulations in all directions in a region near the body extending almost to the analytical stability threshold. Beyond this point, the controller becomes very unstable. Figure 9 shows a typical example $\left(0^{\circ}\right.$ latitude and $-30^{\circ}$ longitude).
These results for hovering above Itokawa have confirmed the qualitative aspects of our findings for body-fixed hovering in the ellipsoidal case subject to both the GDTS w/OL and IATNS controllers. These results suggest that the qualitative properties of the stability regions produced by these controllers can be extended to hovering over realistic asteroid shapes.

\section{Inertial-Frame Hovering}

A large portion of the Hayabusa mission will be dedicated to inertial hovering, during which instrumentation onboard the spacecraft 


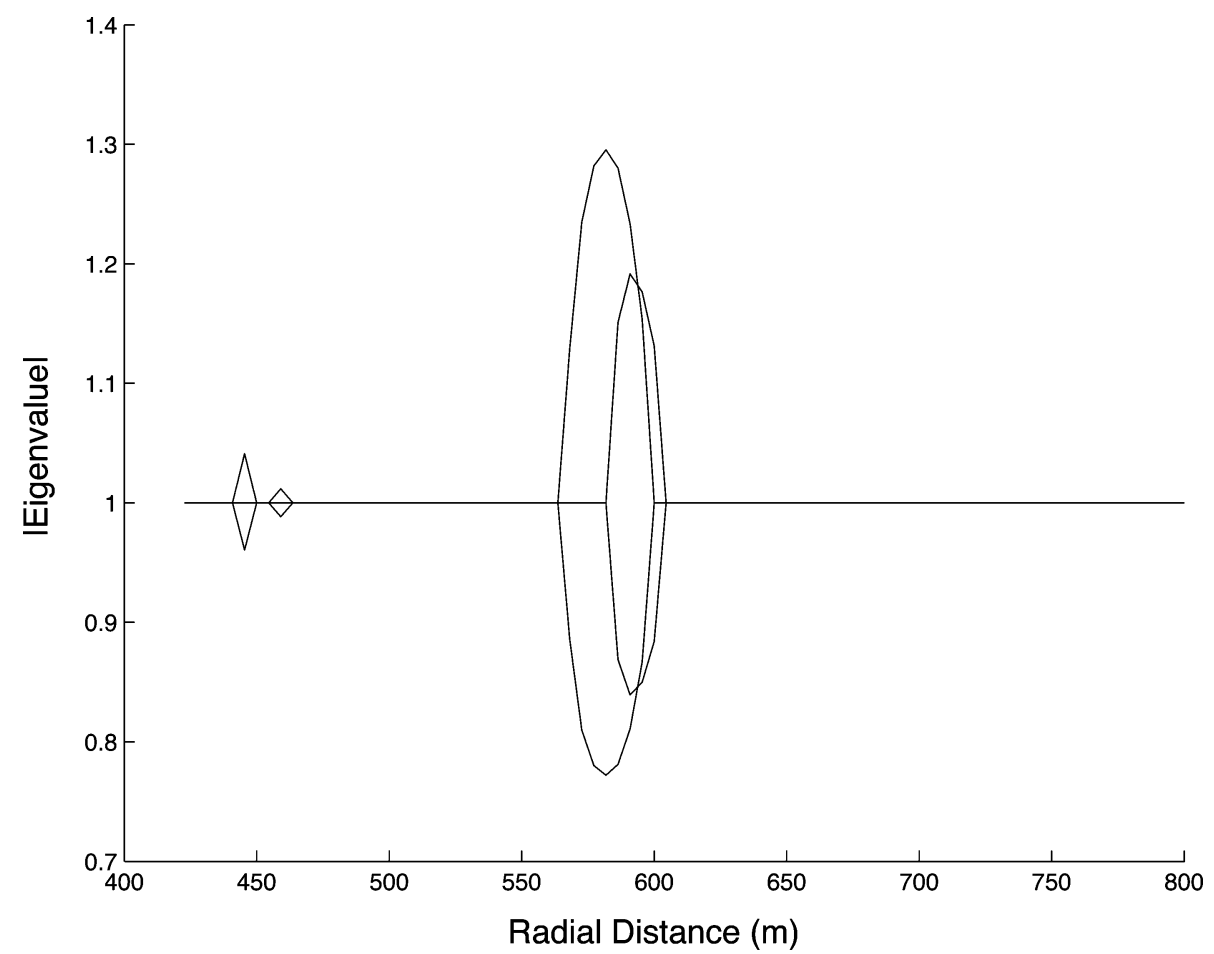

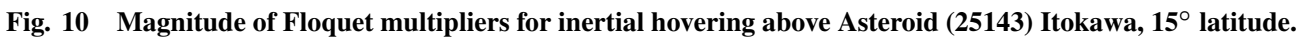

Table 1 Asteroid (25143) Itokawa and Hayabusa mission parameters ${ }^{\mathbf{a}}$

\begin{tabular}{lc}
\hline \hline Parameter & Value \\
\hline Orbital inclination, deg & 1.728 \\
Longitude of ascending node, & 70.921 \\
Orbital eccentricity & 0.280 \\
Orbital period & 1.521 Julian yr \\
Periapsis passage & 2001 May 4.601 \\
Argument of periapsis, deg & 161.029 \\
Orbital semimajor axis, AU & 1.323 \\
Rotation period, h (Ref. 11) & 12.132 \\
Rotation pole, deg (Ref. 12) & $355,-84$ \\
Hayabusa time of arrival (s/c) & $6-15-05$ \\
Hayabusa time of departure (s/c) & $11-2-05$ \\
\hline \hline
\end{tabular}

aCourtesy of "Horizons" Website, ${ }^{13}$ Japan Aerospace Exploration Agency.

will be used to map the asteroid's surface. For this phase of the mission, the Hayabusa mission planners currently intend to implement a controller consisting of three orthogonally oriented position dead-band controllers to maintain spacecraft position. ${ }^{6}$ This type of control effectively forms a "box" in position space that contains the spacecraft's trajectory.

Our previous analysis suggests that the spacecraft can maintain position in inertial space with only a single-dimensional controller at altitudes outside the body's resonance radius. If this is the case, it should result in improved fuel efficiency and operational simplicity for this part of the Hayabusa mission. The current plan is for the Hayabusa spacecraft to hover at an altitude of approximately $20 \mathrm{~km}$ over Itokawa. ${ }^{7}$ Our analysis predicts that inertial hovering at this altitude is stable.

For our results to be as accurate as possible, it was necessary to compute the appropriate latitude at which the spacecraft would be hovering. The spacecraft will be operating in the vicinity of the Earth-asteroid line for purposes of communication. Given the orbit of Itokawa and the appropriate mission data (Table 1) (Refs. 11-13), we calculate that the Earth-asteroid line will move between $14.8^{\circ}$ latitude upon the spacecraft's arrival and $16.8^{\circ}$ at departure. Our analysis is performed at $15^{\circ}$ latitude.
Figure 10 shows the analytical stability results obtained by examining the eigenvalues of the state transition matrix after one period of inertial hovering at $15^{\circ}$ latitude over Itokawa for a range of radial distances. We can see that the primary instability exists in the region near the body's resonance radius of $579 \mathrm{~m}$. Again, we can note that the magnitude of this instability is reasonably small. Interestingly, we see two secondary instabilities near radius $450-\mathrm{m}$. These results are numerically valid (as opposed to the secondary instabilities seen in the ellipsoidal case). Without a more general result, it is difficult to say whether these results validate the idea of secondary harmonic instabilities suggested in the ellipsoidal case or if they exist only because of a specific parameter of this model's shape. Beyond the resonance radius, all inertial hovering is stable. We can conclude that the Hayabusa mission plan to inertially hover at an altitude of $20 \mathrm{~km}$ is safely within the region of stable inertial hovering subject to one-dimensional control when solar gravitation and solar radiation pressure are ignored. The effect of these perturbations will be determined in future work.

\section{Conclusions}

Analytical stability results for hovering in the body-fixed frame were compared to numerical simulation data with realistic hovering control. We found that without the assumption of infinitely tight dead-band control used in the development of the analytical stability conditions, Coriolis accelerations destabilize hovering above the leading edges of ellipsoidal small bodies. The effect of this destabilization depends on the angle between the direction of thrust application and the dead-band orientation. Numerical results for a second realistic controller that uses this knowledge to improve hovering performance near the small body are presented.

Analytical and numerical analyses of hovering in the inertial frame have been performed. We found that inertial hovering is stable at all radii outside the region near the resonance radius up to the point where our two-body assumption breaks down. A shell of unstable inertial hovering positions surrounds the small body at a distance near the body's resonance radius. This instability is strongest in the equatorial plane and strengthens with increasing rotation rates. In most cases, however, the strength of this instability remains mild and it would likely be possible for a spacecraft to move safely across the region. 
The concepts of body-fixed and inertial-frame hovering have been applied to Asteroid (25143) Itokawa, the target of the recently launched Hayabusa mission. Our qualitative results for hovering control above ellipsoidal bodies were found to transfer well to the more general polyhedral shape of Itokawa, suggesting that the control methodologies presented here may be applied to hovering above real small bodies.

\section{Acknowledgments}

The research described in this paper was sponsored by the Interplanetary Network Technology Program by a grant to the University of Michigan from the Jet Propulsion Laboratory, California Institute of Technology, which is under contract with NASA. S. Broschart was also supported by the Graduate Student Researchers Program at the Jet Propulsion Laboratory. The authors thank Shujiro Sawai of the Japan Aerospace Exploration Agency for providing details on the Hayabusa mission.

\section{References}

${ }^{1}$ Scheeres, D. J., Williams, B. G., and Miller, J. K., "Evaluation of the Dynamic Environment of an Asteroid: Applications to 433 Eros," Journal of Guidance, Control, and Dynamics, Vol. 23, No. 3, 2000, pp. 466-475.

${ }^{2}$ Lara, M., and Scheeres, D. J., "Stability Bounds for Three-Dimensional Motion Close to Asteroids," Journal of the Astronautical Sciences, Vol. 50, No. 4, 2002, pp. 389-409.

${ }^{3}$ Scheeres, D. J., and Marzari, F., "Spacecraft Dynamics in the Vicinity of a Comet," Journal of the Astronautical Sciences, Vol. 50, No. 1, 2002, pp. $35-52$.

${ }^{4}$ Scheeres, D. J., "Stability of Hovering Orbits Around Small Bodies," Spaceflight Mechanics 1999: Advances in the Astronautical Sciences, Part II, Vol. 102, 1999, pp. 855-875; also AAS Paper 99-159, 1999.
${ }^{5}$ Sawai, S., Scheeres, D. J., and Broschart, S. B., "Control of Hovering Spacecraft Using Altimetry," Journal of Guidance, Control, and Dynamics, Vol. 25, No. 4, 2002, pp. 786-795.

${ }^{6}$ Kubota, T., Hashimoto, T., Uo, M., Maruya, M., and Baba, K., "Maneuver Strategy for Station Keeping and Global Mapping Around an Asteroid," Spaceflight Mechanics 2001: Advances in the Astronautical Sciences, Vol. 108, 2001, pp. 769-779; also AAS Paper 01-156. 2001.

${ }^{7}$ Hashimoto, T., Kubota, T., Kawaguchi, J., Uo, M., Baba, K., and Yamashita, T., "Autonomous Descent and Touch-down via Optical Sensors," Spaceflight Mechanics 2001: Advances in the Astronautical Sciences, Vol. 108, 2001, pp. 469-490; also AAS Paper 01-134, 2001.

${ }^{8}$ Scheeres, D. J., "Dynamics About Uniformly Rotating Tri-Axial Ellipsoids: Applications to Asteroids," Icarus, Vol. 110, No. 2, 1994, pp. 225-238.

${ }^{9}$ Werner, R. A., and Scheeres, D. J., "Exterior Gravitation of a Polyhedron Derived and Compared with Harmonic and Mascon Gravitation Representations of Asteroid 4769 Castalia," Celestial Mechanics and Dynamical Astronomy, Vol. 65, No. 3, 1996, pp. 313-344.

${ }^{10}$ Kawaguichi, J., Kuninaka, H., Fujiwara, A., Uesugi, T., and Ohnishi, T., "MUSES-C Launch and Early Operations Report," AAS Paper 03-662, Aug. 2003.

${ }^{11}$ Ostro, S. J., Benner, L. A. M., Nolan, M. C., Magri, C., Giorgini, J. D., Scheeres, D. J., Broschart, S. B., Kaasalainen, M., Vokrouhlicky, D., Chesley, S. R., Margot, J., Jurgens, R. F., Rose, R., Yeomans, D. K., Suzuki, S., and De Jong, E.M., "Radar Observations of Asteroid 25143 Itokawa (1998 SF36)," Meteoritics and Planetary Science, Vol. 39, No. 3, 2004, pp. 407-424.

${ }^{12}$ Kaasalainen, M., Kwiatkowski, T., Abe, M., Piironen, J., Nakamura, T., Ohba, Y., Dermawan, B., Farnham, T., Colas, F., Lowry, S., Weissman, P., Whiteley, R. J., Tholen, D. J., Larson, S. M., Yoshikawa, M., Toth, I., and Velichko, F. P., "CCD Photometry and Model of MUSES-C Target (25143) 1998 SF36," Astronomy and Astrophysics, Vol. 405, 2003, pp. L29-L32.

13“"JPL's Horizons System," URL: http://ssd.jpl.nasa.gov/horizons.html [cited 15 Dec. 2003]. 